\title{
Prognostic significance of metadherin overexpression in hepatitis B virus-related hepatocellular carcinoma
}

\author{
ZHENBIN GONG $^{1,2^{*}}$, WEIHUI LIU ${ }^{1 *}$, NAN YOU $^{1 *}$, TAO WANG $^{1}$, XING WANG $^{1}$, \\ PENG LU $^{1}$, GE ZHAO ${ }^{1}$, PING YANG ${ }^{1}$, DESHENG WANG ${ }^{1}$ and KEFENG DOU ${ }^{1}$ \\ ${ }^{1}$ Department of Hepatobiliary Surgery, Xijing Hospital, Fourth Military Medical University, Xi'an 710032; \\ ${ }^{2}$ Center of Hepatobiliary Surgery of Lanzhou Army Region, Lanzhou 730000, P.R. China
}

Received December 3, 2011; Accepted February 23, 2012

DOI: $10.3892 / o r .2012 .1749$

\begin{abstract}
Metadherin (MTDH), which is an HIV-1 or TNF- $\alpha$-inducible transcript, has a crucial role in several types of cancer by regulating multiple cellular signaling processes. However, to date, the role of MTDH in hepatitis $\mathrm{B}$ virus (HBV)-related hepatocellular carcinoma (HCC) is still unclear. In the present study, we detected MTDH expression in normal liver, chronic hepatitis B and HBV-related HCC tissues. The data showed that MTDH expression levels were elevated in the hepatitis B tissues and especially in the HBV-related HCC tissues compared to normal liver tissues. There was a trend for gradually increased MTDH expression from normal liver tissue to hepatitis B and HBV-related HCC tissues. Furthermore, a statistical analysis revealed that MTDH expression significantly correlated with the American Joint Committee on Cancer (AJCC, 7th edition) stage $(\mathrm{P}=0.020), \mathrm{T}$ classification $(\mathrm{P}=0.007), \mathrm{N}$ classification $(\mathrm{P}=0.044)$, vascular invasion $(\mathrm{P}=0.006)$ and histological differentiation $(\mathrm{P}=0.020)$ in the HBV-related HCC patients. In addition, patients with high MTDH levels had shorter survival times compared to those with low MTDH expression $(\mathrm{P}=0.001)$. Taken together,
\end{abstract}

Correspondence to: Dr Kefeng Dou or Dr Desheng Wang, Department of Hepatobiliary Surgery, Xijing Hospital, Fourth Military Medical University, Xi'an 710032, P.R. China

E-mail: doukf2008@yahoo.cn

E-mail: wangds@fmmu.edu.cn

*Contributed equally

Abbreviations: AEG-1, astrocyte elevated gene-1; AFP, $\alpha$-fetoprotein; AJCC, American Joint Committee on Cancer; AP-1, activator protein 1; ERK, extracellular signal-regulated kinase; GSK-3 $\beta$, glycogen synthesis kinase-3 $\beta$; HBV, hepatitis B virus; $\mathrm{HBx}$, hepatitis B virus X protein; $\mathrm{HCC}$, hepatocellular carcinoma; HIV-1, human immunodeficiency virus type 1; MTDH, metadherin; NF- $\mathrm{B}$, nuclear factor-kappa B; PI3K, phosphoinositide 3-kinase; TNF- $\alpha$, tumor necrosis factor- $\alpha$

Key words: metadherin, hepatocellular carcinoma, hepatitis B virus, progression, prognosis these results suggest that MTDH could be a potential prognostic marker for overall survival and tumor progression and a chemotherapeutic target in HBV-related HCC patients.

\section{Introduction}

Hepatocellular carcinoma (HCC) is the fifth most common cancer and the third most common cause of cancer mortality worldwide (1,2). Since the 1990s, it has been ranked the second leading cause of cancer deaths in China. Epidemiological studies have identified major risk factors for HCC, including infection with hepatitis $\mathrm{B}$ and $\mathrm{C}$ virus ( $\mathrm{HBV}$ and $\mathrm{HCV}$ ), exposure to aflatoxin B1, a high intake of alcohol, as well as metabolic diseases (2). Among these risk factors, growing evidence supports $\mathrm{HBV}$ infection as the most common cause of HCC. According to a recent study, 53\% of HCC cases worldwide are associated with HBV infection (3). Because of endemic HBV infection, HBV-related HCC has a higher prevalence in China than in Europe and America.

HBx protein, which is coded by the HBV X gene (HBX gene), is frequently integrated with the HBV genome into cellular DNA during hepatocellular carcinogenesis and remains functionally active, regulates cell cycle progression (4), promotes cell proliferation, inhibits cell apoptosis (5), and the expression of several tumor suppressor genes including p53 (4) and senescence-related factors. However, the exact molecular mechanisms and pathways responsible for HBV-related HCC are still unclear. Therefore, it is important to elucidate the key factors that are involved in HBV-related HCC, which will help to identify HBV-related HCC patients with elevated risk, improve prognosis prediction and facilitate therapeutic intervention.

The oncoprotein metadherin (MTDH, also known as astrocyte elevated gene-1, AEG-1) was originally identified as a protein that is induced in primary human fetal astrocytes infected with human immunodeficiency virus-1 (HIV-1) or treated with HIV gp120 or tumor necrosis factor- $\alpha$ (TNF- $\alpha$ ) (6). Overexpression of MTDH has been frequently observed in a variety of primary human tumors, including breast cancer (7-10), multiform glioblastoma (11-13), neuroblastoma $(14,15)$, gastric cancer (16), colorectal carcinoma (17), osteosarcoma (18), gallbladder carcinoma (19), head and neck squamous cell carcinoma (HNSCC) (20), endometrial cancer (21), ovarian 
carcinoma (22), esophageal squamous cell carcinoma (ESCC) (23), non-small cell lung cancer (24), HCC (25), melanoma (26), and prostate cancer $(6,27,28)$. Furthermore, published studies on some cancers have revealed that MTDH is a novel and useful prognostic marker for cancer progression, and its overexpression is associated with an unfavorable prognosis $(7,8,13,15-17,23,24,26,28,29)$. The role of MTDH in HCC has long been debated. A previous study showed that MTDH is a key regulator of multiple facets that are critical for HCC development (25). However, the role of MTDH in HBV-related HCC remains unclear. The data that have accumulated over the last few years suggest that MTDH could regulate the transformed status of some types of cancer through the activation of different signaling molecules, which overlap with the signaling pathways associated with $\mathrm{HBx}$. Considering these data, we believe that MTDH may be closely linked to HBV-related HCC and should be further investigated.

In this study, we investigated the expression of MTDH in HBV-related HCC patients and the correlation between MTDH expression and clinicopathological characteristics and survival. To our knowledge, this is the first report to describe the correlation between MTDH and HBV-related HCC. The expression of MTDH was upregulated in the surgically extracted tissues of HBV-related HCC and hepatitis B patients. Moreover, the overexpression of MTDH significantly correlated with the American Joint Committee on Cancer (AJCC, 7th edition) stage, tumor-node-metastasis (TNM) classification and the histological classification of HBV-related HCC. More importantly, a multivariate analysis revealed that MTDH could be an independent biomarker for the prediction of HBV-related HCC prognosis. These results support a novel role for MTDH as an essential intermediary in HBV-related HCC and suggest that MTDH could be a useful clinical biomarker and therapeutic target in HBV-related HCC.

\section{Materials and methods}

Patients and tissue samples. Clinical samples of HBV-related HCC and hepatitis B tissues were obtained from 73 patients with HBV-related HCC and 45 patients with hepatitis B who underwent initial radical hepatectomy and liver biopsy at the Xijing Hospital of the Fourth Military Medical University (Xi'an, Shaanxi, China) from July 2004 to June 2006. Normal liver tissues were obtained from 11 patients who underwent hepatectomy due to benign liver disease. The study was approved by the Xijing Hospital Ethics Committee, and informed consent was obtained from all of the patients. The recommendations of the Declaration of Helsinki for biomedical research involving human subjects were followed. The patients with HBV-related HCC in this study included 62 male and 11 female patients with a median age of 54 years (range, 38.072.0 years). None of the patients had received chemotherapy or radiation therapy prior to surgery. The clinicopathological parameters of the patients are shown in Table II. All of the diagnoses of HBV-related hepatitis and HCC were confirmed by the serological detection of HBV antigens and were verified by pathologists. Tumor differentiation grades were determined according to the Edmondson-Steiner classification system. Using the AJCC (7th edition) classification, the patients with HCC were classified as stages I, II, III and IV. There were 48 patients with stage I, 9 with stage II, 8 with stage III, and 8 with stage IV. Overall survival was calculated as the time from the date of the operation until death. The follow-up of the patients was regularly performed at outpatient clinics. $\alpha$-fetoprotein (AFP) assay and liver sonography were performed every 3 months, if necessary, and computerized tomography (CT) was also performed.

Immunohistochemical staining. The formalin-fixed and paraffin-embedded tissue specimens included HCC $(n=73)$, hepatitis $B(n=45)$ and normal liver $(n=11)$ tissues. The procedures were carried out as described in the instruction manual. In brief, paraffin-embedded specimens were cut into $4 \mu \mathrm{m}$ sections and baked at $60^{\circ} \mathrm{C}$ for $60 \mathrm{~min}$. After deparaffinization with xylene and rehydration with graded alcohol, the sections were rinsed in phosphate-buffered saline (PBS). Subsequently, the slides were placed in citrate buffer and were microwaved on the medium setting for $5 \mathrm{~min}$. The sections were treated with $3 \%$ hydrogen peroxide in methanol for $20 \mathrm{~min}$ at room temperature to suppress the endogenous peroxidase activity and were incubated with pre-immune serum albumin to block non-specific binding. Anti-MTDH (1:400, rabbit polyclonal, Proteintech Group, Inc., Chicago, IL, USA) was incubated with the sections overnight at $4^{\circ} \mathrm{C}$. For the negative controls, the anti-MTDH antibody was replaced with goat serum in this step. After washing with TBST buffer, the tissue sections were treated with a biotinylated secondary antibody followed by an incubation with streptavidin-horseradish peroxidase complex. The tissue sections were then immersed in DAB and counterstained with $10 \%$ Mayer's hematoxylin to visualize the staining. Finally, the slides were dehydrated and mounted.

Immunohistochemical assessment. The degree of immunostaining for protein expression in the specimens was observed and scored separately by three independent investigators, who were blinded to the patient information. At x200 magnification, 10 representative fields of each section were viewed. The sections were scored by combining the proportion of positively stained cells with the intensity of the staining. The scores obtained by the three independent investigators were averaged for the comparative evaluation of MTDH expression. The cell proportion was scored as follows: 0 , no positive cells; $1,<10 \%$ positive cells; $2,<50 \%$ positive cells; and $3,>50 \%$ positive cells. The staining intensity was graded using the following criteria: 0 (no staining); 1 (weak staining = light yellow); 2 (moderate staining $=$ yellow brown); and 3 (strong staining = brown). The staining index (SI) was calculated as the product of the staining intensity score and the proportion of positive tumor cells. Using this method of assessment, we evaluated MTDH expression in the sections by calculating SI scores of $0,1,2,3,4,6$ or 9 . Based on the measure of heterogeneity from the log-rank test, an optimal cut-off value for high and low expression level was identified: an SI score of $\geq 6$ was used to define tumors with high MTDH expression, and an SI score of $\leq 4$ was used to indicate low MTDH expression.

Western blotting. Total proteins of HCC (10 cases), hepatitis B (10 cases), and normal (10 cases) tissues were obtained from the patients previously mentioned. The protein concentrations were quantified, and $40 \mu \mathrm{g}$ of protein per sample was separated 
using sodium dodecyl sulfate polyacrylamide gel electrophoresis and transferred to a polyvinylidene fluoride membrane (Immobilon-P; Millipore, Bedford, MA, USA), which had been soaked in $100 \%$ methanol for 2 min. After blocking with $5 \%$ non-fat dry milk, the membranes were incubated with anti-MTDH (1:400, rabbit polyclonal, Proteintech Group) for $1 \mathrm{~h}$ at room temperature. After washing with TBST buffer, the membranes were incubated with a horseradish peroxidaseconjugated secondary antibody for $1 \mathrm{~h}$ at room temperature. Finally, the membranes were covered with enhanced chemiluminescence reagents (Applygen Technologies Inc., Beijing, China) for $1 \mathrm{~min}$ and were exposed to the film to visualize the result. The experiment was performed in triplicate. Western blotting for $\beta$-actin was used as an internal loading control.

RNA extraction, reverse transcription and real-time PCR. Total RNA was extracted from the surgically obtained HCC, hepatitis $\mathrm{B}$ and normal liver tissues using TRIzol reagent (Invitrogen, Carlsbad, CA, USA) according to the manufacturer's instructions. The cDNA was synthesized using the Primescript RT Reagent kit (Takara, Dalian, China) using the oligo(dT) primer. The qRT-PCR analysis was performed with the SYBR Premix EX Taq II (Takara) on a Bio-Rad IQ5 (BioRad Laboratories, Hercules, CA, USA) machine. All of the specific primers used in the present study were designed and synthesized by Sangon Biotech (Shanghai, China). The housekeeping gene glyceraldehyde 3-phosphate dehydrogenase (GAPDH) was selected as a reference gene. The sequences of the primers were as follows: MTDH, 5'-AAGAGGAAAACT GAGCCATCTG-3' (forward) and 5'-CGGCTAACATCCCA CTGATAAT-3' (reverse); and GAPDH, 5'-GACTCATGAC CACAGTCCATGC-3' (forward) and 5'-AGAGGCAGGGAT GATGTTCTG-3' (reverse). Each reaction was performed in triplicate. The specific reaction conditions were as follows: initial denaturation at $95^{\circ} \mathrm{C}$ for $1 \mathrm{~min}$; template denaturation at $95^{\circ} \mathrm{C}$ for $20 \mathrm{sec}$, annealing at $60^{\circ} \mathrm{C}$ for $30 \mathrm{sec}$, and extension at $72^{\circ} \mathrm{C}$ for $1 \mathrm{~min}$ (total of 40 cycles) and final extension at $72^{\circ} \mathrm{C}$ for $10 \mathrm{~min}$. In a control reaction, cDNA was substituted with water.

Statistical analysis. The $\chi^{2}$ test and Fisher's exact test were used to analyze categorical variables. Bivariate correlations between study variables were calculated by Spearman's rank correlation coefficients. Survival curves were plotted by the Kaplan-Meier method and the difference was compared using the log-rank test. Prognostic relevance was evaluated using a multivariate Cox regression analysis. All of the statistical analyses were performed using the SPSS 17.0 software (SPSS Inc., Chicago, IL). $\mathrm{P}<0.05$ was considered statistically significant.

\section{Results}

Differential expression of $M T D H$. Western blotting revealed that the MTDH protein was differentially upregulated in all of the hepatitis B and HCC tissues. In contrast, it was not detected in normal liver tissues $(\mathrm{P}<0.001$, Fig. 1A and B). Similarly, qRT-PCR results showed that all of the HBV-related HCC tissues exhibited significantly (up to 10-fold) higher levels of MTDH messenger RNA compared with normal liver tissues (Fig. 1C).
$\mathbf{A}$

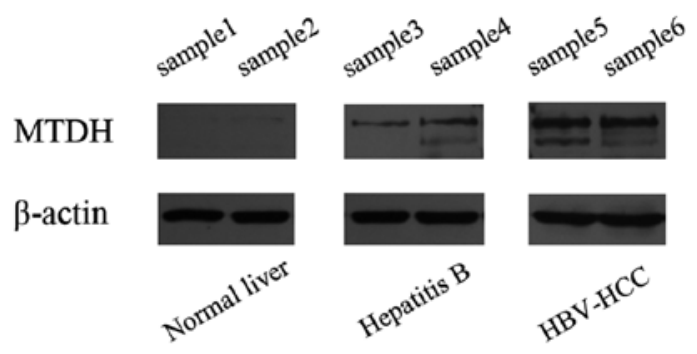

B
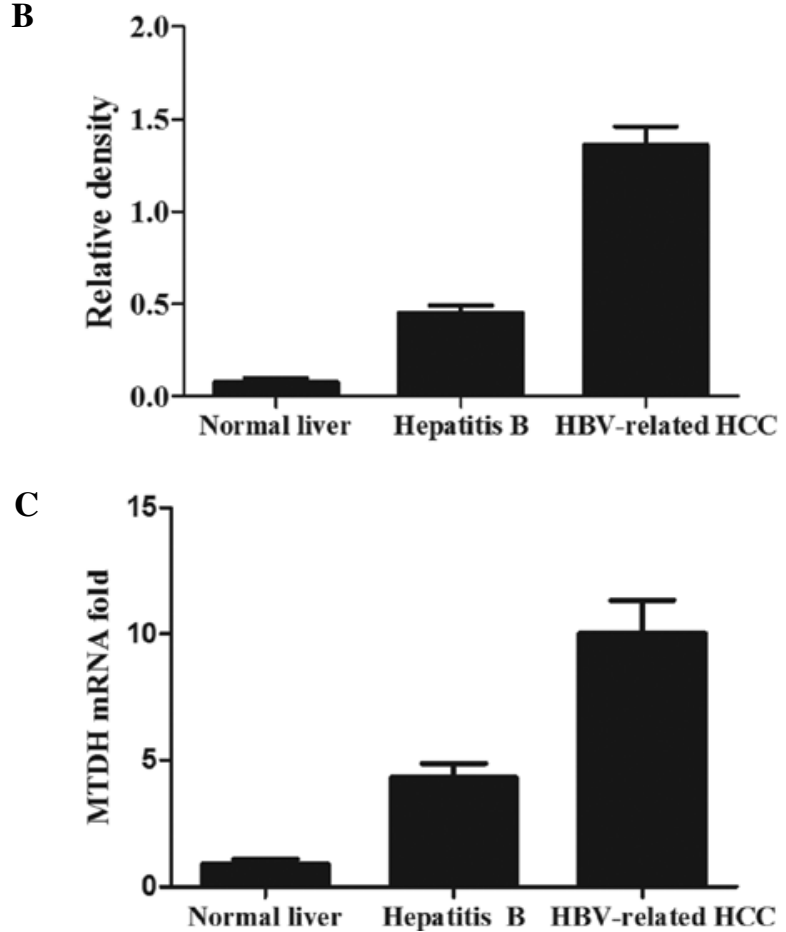

Figure 1. MTDH protein and mRNA levels in different liver tissues. (A) Western blotting shows that MTDH expression is upregulated in HBV-related hepatitis and HCC, whereas weak or no MTDH expression was observed in normal liver tissue. Expression levels were standardized against $\beta$-actin. (B) MTDH protein expression levels in the individual tissue samples was calculated as the ratio of MTDH expression relative to $\beta$-actin expression (mean $\pm \mathrm{SD}, \mathrm{P}<0.001)$. (C) qRT-PCR assay of MTDH mRNA expression in normal liver, HBV-related hepatitis and HCC tissues (mean $\pm \mathrm{SD}, \mathrm{P}<0.001$ ). Expression levels were normalized to GAPDH.

Using immunohistochemical staining, very little to no MTDH was detected in normal liver specimens, whereas significant MTDH staining was observed in the hepatitis B and HBV-related HCC samples. MTDH expression was mainly localized in the cytoplasmic region, and nuclear MTDH staining was not detected in any sections. There were 2 out of 45 hepatitis B and 3 out of 73 HBV-related HCC samples that scored zero for MTDH. No significant difference was found between hepatitis $\mathrm{B}$ patients with cirrhosis and those without cirrhosis $(\mathrm{P}=0.139)$. The frequency and intensity of MTDH expression was gradually elevated from normal liver tissues to hepatitis B, with the highest indices in HBV-related HCC (Table I; Fig. 2).

Relationship between MTDH expression and the clinicopathological parameters of $H B V$-related $H C C$. As shown in Table II, MTDH expression significantly correlated with the AJCC stage $(\mathrm{P}=0.020)$, T classification $(\mathrm{P}=0.007)$, $\mathrm{N}$ classification $(\mathrm{P}=0.044)$, vascular invasion $(0.006)$, and histological 
Table I. Immunohistochemical analysis of MTDH in different liver tissues.

\begin{tabular}{llcl}
\hline & \multicolumn{2}{c}{ MTDH expression } & \\
\cline { 2 - 3 } Categories & Low $(\%)$ & High (\%) & P-value \\
\hline Normal liver tissue & $11(100)$ & $0(0)$ & $<0.001$ \\
Hepatitis B & $13(53.3)$ & $9(46.7)$ & \\
HBV-related HCC & $26(35.6)$ & $47(64.4)$ & \\
\hline
\end{tabular}

P-values were calculated by the $\chi^{2}$ test and Fisher's exact test.

differentiation $(\mathrm{P}=0.020)$ of patients with HBV-related HCC. For the $\mathrm{M}$ classification $(\mathrm{P}=0.083)$, all of the 6 patients with M1 exhibited high MTDH expression. To further confirm the correlation between MTDH expression and clinicopathological features, a Spearman correlation analysis was used. Table III shows the Spearman correlations of MTDH expression levels to histological differentiation $(0.274 ; \mathrm{P}=0.019)$, AJCC stage $(0.285 ; \mathrm{P}=0.015)$, and $\mathrm{T}$ classification $(0.244 ; \mathrm{P}=0.048)$.

Association between MTDH expression and the prognosis of patients with HCC. Statistical analyses showed a significant negative correlation between the MTDH staining index and the overall survival time of HBV-related HCC patients
(Spearman correlation coefficient -0.385; $\mathrm{P}=0.001$ ). A KaplanMeier analysis and the log-rank test were applied to calculate the effect of MTDH expression on overall patient survival. The log-rank test revealed that the MTDH expression level correlated significantly with the survival time of patients with HCC $(\mathrm{P}=0.001)$. Furthermore, the median survival time of patients with high MTDH expression levels was 30 months (95\% confidence interval, 23.4-36.8 months), while the median survival time of those with low expression was 49 months $(95 \%$ confidence interval, 42.5-52.8 months). The 5-year cumulative survival rate was $65.1 \%$ in the low MTDH expression group, whereas it was only $19.4 \%$ in the high MTDH expression group (Fig. 3).

To determine whether the MTDH expression level is an independent prognostic factor in patients with $\mathrm{HCC}$, we performed a multivariate survival analysis as described in Materials and methods. Our findings revealed that MTDH expression, AJCC stage, T classification, and tumor differentiation are independent prognostic factors (Table IV). Taken together, these results indicate that MTDH expression has a significant correlation with the prognosis of HBV-related HCC.

\section{Discussion}

Despite remarkable advances in diagnosis and therapeutic techniques, only a minority of patients with HCC are diagnosed at early stages and are candidate for curative treatments, and the recurrence rate may be as high as $50 \%$ at 3 years (1).
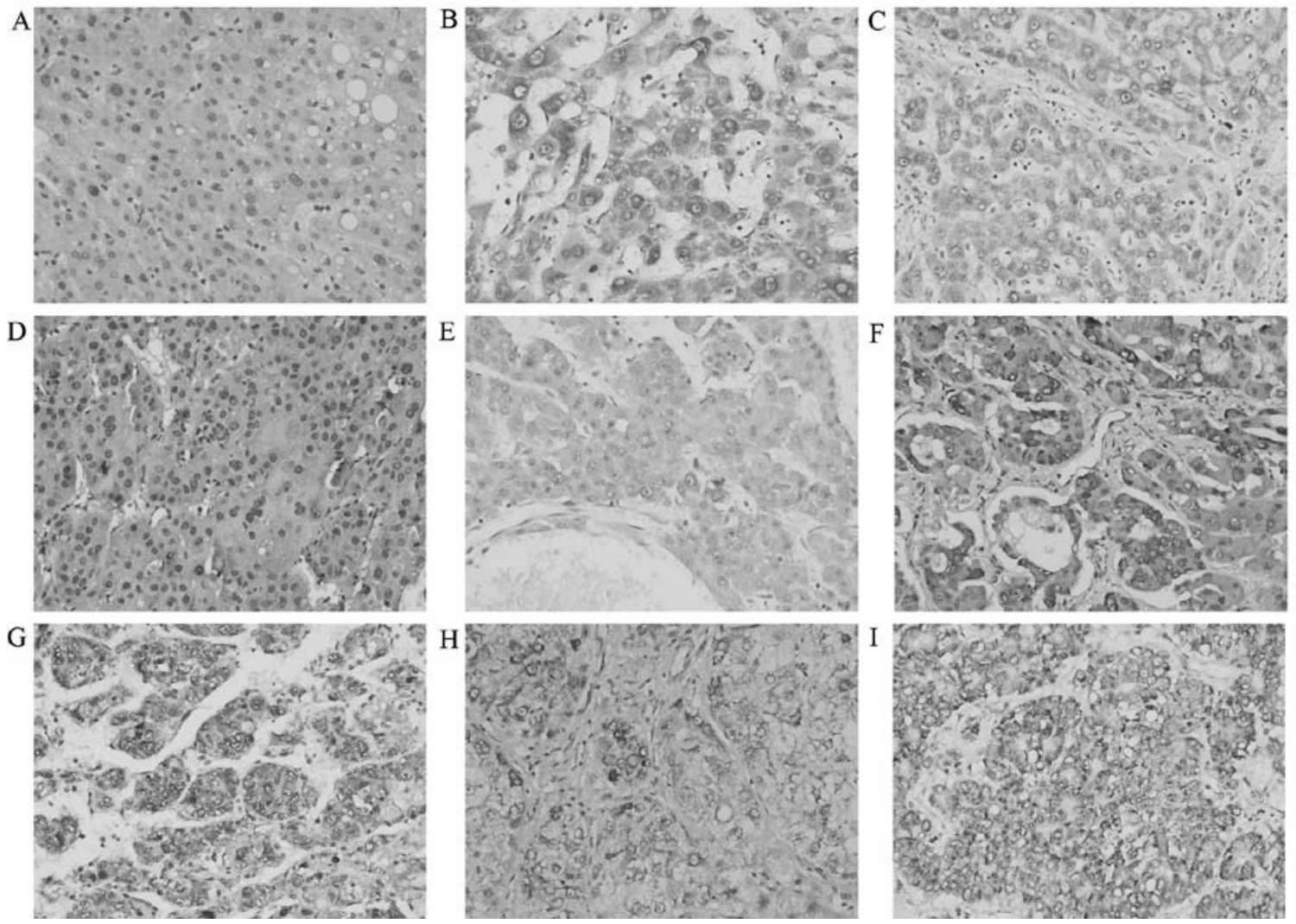

Figure 2. Immunohistochemical staining of MTDH in different liver tissues. (A) Normal liver tissues; (B) hepatitis B without cirrhosis; (C) hepatitis B with cirrhosis; (D) HBV-related HCC, scored zero; (E) HBV-related HCC, stage I, good differentiation; (F) HBV-related HCC, stage I, moderate differentiation; (G) HBV-related HCC, stage II, poor differentiation; (H) HBV-related HCC, stage III, moderate differentiation. (I) HBV-related HCC, stage IV, moderate differentiation. Original magnification, $\mathrm{x} 400$. 
Table II. Relationship between MTDH expression and clinicopathological parameters in patients with HBV-related HCC.

\begin{tabular}{lllll}
\hline & $\begin{array}{c}\text { MTDH } \\
\text { expression }\end{array}$ & & \\
\cline { 2 - 5 } Parameters & Low High & n & P-value
\end{tabular}

Age (years)

$$
\leq 54
$$$$
>54
$$

14

12

0.463

Gender

Male

Female

Tumor size $(\mathrm{cm})$

$\leq 5$

$>5$

Tumor number

$$
\leq 2
$$$$
>2
$$

AJCC stage

$$
\text { I }
$$

II

III

IV

\section{$\mathrm{T}$ classification}

$$
\text { T1 }
$$

T2

T3

T4

$\mathrm{N}$ classification

$$
\mathrm{N} 1
$$

No

M classification

M1

M0

Histological differentiation

$$
\text { Good }
$$

Poor

Vascular invasion

$$
\begin{aligned}
& \text { Absence } \\
& \text { Presence }
\end{aligned}
$$

Serum AFP levels $(\mu \mathrm{g} / \mathrm{l})$

$\begin{array}{rrrr}\leq 400 & 22 & 29 & 51 \\ >400 & 4 & 18 & 22\end{array}$

${ }^{\mathrm{a}} \mathrm{P}<0.05$ was considered as statistically significant ( $\mathrm{P}$-values were calculated using the $\chi^{2}$ test and the Fisher's exact test).

In contrast, the remaining patients face extremely poor prognoses. It is believed that the main cause of the extremely poor prognoses for patients with HCC is the fact that the molecular

0.062
Table III. Spearman correlation analysis between MTDH expression and clinicopathological parameters.

\begin{tabular}{lc}
\hline Values & Spearman correlation (P-value) \\
\hline Histological differentiation & $0.274(0.019)$ \\
AJCC stage & $0.285(0.015)$ \\
T classification & $0.244(0.048)$ \\
\hline
\end{tabular}

Table IV. Multivariate analysis of various prognostic variables in patients with $\mathrm{HBV}$-related HCC

\begin{tabular}{lcc}
\hline Variable & $\begin{array}{c}\text { Relative risk } \\
(95 \% \text { confidence interval })\end{array}$ & P-value \\
\hline MTDH expression & $7.314(1.848-28.398)$ & 0.004 \\
AJCC stage & $3.090(1.468-6.505)$ & 0.003 \\
T classification & $1.871(0.928-3.773)$ & 0.080 \\
Tumor differentiation & $2.321(1.131-4.764)$ & 0.022 \\
\hline
\end{tabular}

The P-values were calculated using a Cox-regression analysis.

\section{Overall survival}

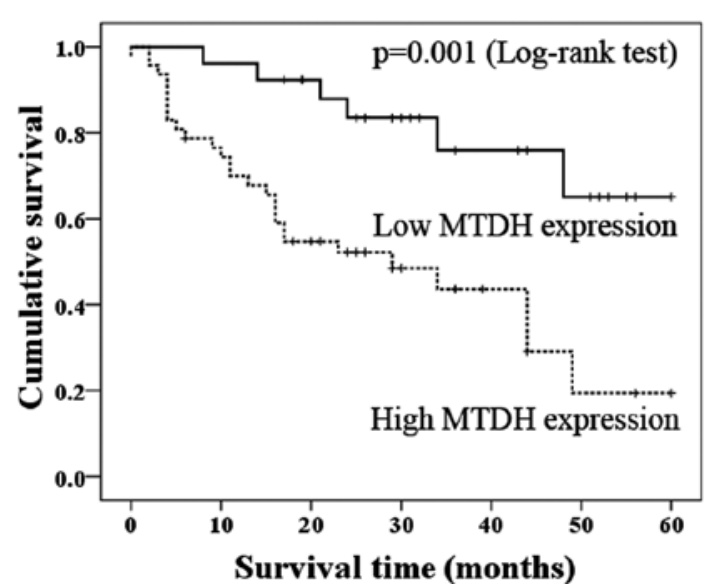

Figure 3. Kaplan-Meier curve showing the relationship between MTDH expression and the survival time of patients with HBV-related HCC. The cumulative 5-year survival rates in the high MTDH expression group and the low MTDH expression group were $19.4 \%$ and $65.1 \%$, respectively (log-rank $\mathrm{P}=0.001)$.

mechanisms responsible for HCC are unclear. In China, chronic $\mathrm{HBV}$ infection has been identified as a major risk factor for $\mathrm{HCC}$, and the prognosis of patients with HBV-related HCC is very poor. Therefore, elucidating the molecular mechanism and identifying prognostic and therapeutic targets are important for HBV-related HCC. In this study, we primarily focused on HBV-related HCC.

The molecular mechanisms responsible for the pathogenesis of HBV-related HCC are completely different from those of HCV-related HCC (30). Previous research has confirmed that the $\mathrm{HBx}$ protein of $\mathrm{HBV}$ has a crucial role in the pathogenesis of HBV-related HCC and activates several different 
signaling pathways that are involved in HCC, including the JAK/STAT (31), Wnt/ $\beta$-catenin $(32,33), \mathrm{RAS} / \mathrm{RAF} / \mathrm{MAPK}$, and PI3K/AKT/ERK pathways and their downstream molecules, such as GSK-3 $\beta$, cyclin D, A and B, and p21 $1^{\text {cip1 }}$ (34-36). Additionally, $\mathrm{HBx}$ has been shown to activate the transcription factors NF- $\mathrm{B}$ (37) and AP-1 (38). However, the exact molecular mechanisms responsible for HBV-mediated hepatocarcinogenesis are incompletely understood. Furthermore, the antiviral treatment of $\mathrm{HBV}$ infection may render patients with HBV-related HCC better able to tolerate HCC treatments and may improve prognosis. However, it is important to note that conventional treatment, such as lamivudine, inhibits $\mathrm{HBV}$ replication but cannot reverse HBV-related changes in downstream signaling pathways, which are closely related to the occurrence and development of HCC (39). Considering the above findings, we believe that it is important to investigate the direct molecular mechanism of the carcinogenic effect of HBV and to identify new intermediaries. The results of these studies may help to identify additional therapeutic targets for HBV-related HCC.

MTDH, which is a key regulator of multiple critical facets for the development of malignancy, has been demonstrated to inhibit cancer cell apoptosis and to increase invasiveness and metastasis. Meanwhile, the knockdown of MTDH has the inverse effects $(28,40)$. In addition, researchers have identified a lung-homing domain of MTDH, which mediates breast cancer cell metastasis to the lung by adhering to the lung vasculature (10). Furthermore, MTDH regulates different signaling pathways that are closely related to cancer, such as NF- $\kappa \mathrm{B}(27,41,42), \mathrm{Wnt} / \beta$-catenin (25), MAPK/ERK (40), PI3K/ AKT $(26,40)$, and AP-1 (28). All of these studies indicate that MTDH is potentially a key mediator of cancer and a crucial part of oncogenic signaling networks. Most importantly, many of the pathways that have been associated with MTDH overlap with the signaling pathways regulated by HBx. Therefore, we postulated that MTDH may be required for the development of HBV-related HCC.

In the present study, we found for the first time that MTDH protein was strongly overexpressed in HBV-related HCC tissue samples by western blotting. However, it was weak in hepatitis B tissues and was not detected in normal hepatic tissues, which indicates a gradient expression model. Immunohistochemical staining revealed a similar gradual elevation model. MTDH staining was mainly localized in the cytoplasm of cells, similarly to the reports of Yoo et al (25). Because MTDH overexpression has also been detected in colorectal adenoma (17) and breast ductal carcinoma in situ (43), we have reasons to believe that MTDH overexpression might be an early event during carcinogenesis. Therefore, MTDH could be used as an early-stage diagnostic marker in HBV-related HCC. Additionally, the positive expression of MTDH in the hepatitis B tissue samples suggested that one of the reasons for MTDH activation during the initiation of HBV-related HCC may be HBV infection. Of course, this conclusion should be confirmed in further studies.

A statistical analysis of the relationship between MTDH staining and the clinical parameters of patients with HBV-related HCC showed a significant correlation of MTDH expression with AJCC stage, histological differentiation, vascular invasion, $\mathrm{T}$, and $\mathrm{N}$ classifications. MTDH expression was gradually elevated with the loss of cancer differentiation and with the elevation of AJCC stages. Moreover, the cumulative 5-year survival rate of patients in the high MTDH expression group was significantly lower than that of patients in the low MTDH expression group. A multivariate analysis verified that MTDH expression is an independent prognostic factor of patients with HBV-related HCC. MTDH expression status may be correlated with carcinogenesis, progression and poor prognosis of $\mathrm{HBV}$-related HCC, similarly to published studies in other types of cancer $(7,9,10,15,23,24,26,28)$. All of these results strongly indicated that MTDH could be utilized as a novel biomarker to identify the progression of HBV-related HCC and as a predictor for patient prognosis and survival. The data from our study partly confirmed the observation by Yoo et al (25), who reported a significant association between MTDH and HCC. However, their study did not reveal a significant relationship between the MTDH expression levels and HBV-related HCC. Therefore, it could be said that our data show further proof of the involvement of MTDH in HCC. We demonstrated that MTDH is a very important mediator for $\mathrm{HBV}$ infection to induce HCC.

It is worth noting that some studies have reported that the inhibition of MTDH may sensitize tumor cells to current antitumor treatments. It was demonstrated that MTDH enhances the expression of dihydropyrimidine dehydrogenase (DPYD), which catalyzes the initial and rate-limiting step in the catabolism of 5-FU, which is an adjuvant and palliative chemotherapeutic agent that is frequently used in the treatment of patients with HCC (44). In addition, MTDH augments the expression of the transcription factor LSF, which regulates the expression of thymidylate synthase (TS), a target of 5-FU. A similar study found that knockdown of MTDH expression in human neuroblastoma cells significantly enhanced chemosensitivity to cisplatin or doxorubicin $(15,41)$. In addition, using lentiviral delivery of MTDH siRNA in combination with 5-FU more effectively inhibited the growth of human HCC cells xenotransplanted in athymic nude mice than either agent alone (44). These results suggest that MTDH siRNA could be a novel adjuvant therapy of HCC.

Our study is among the first to demonstrate that MTDH may be required for $\mathrm{HBV}$-related $\mathrm{HCC}$. However, a better understanding of the HBV-MTDH signaling pathways remains a high priority for future investigation. In conclusion, these results suggest that MTDH could be effective as both a clinical indicator of progression and a prognostic biomarker for survival in patients with HBV-related HCC. MTDH may also be a molecular target for new anticancer agents to prevent HBV-related HCC progression and metastasis. In addition, according to our findings, HBV may have a role as an initiator of MTDH activation during the initiation of HBV-related HCC. We will verify these predictions and elucidate the molecular mechanism of the effects of MTDH in the progression of $\mathrm{HBV}$-related HCC in future studies.

\section{Acknowledgements}

The authors would like to thank Dr Jiangang Long for his excellent technical assistance and Dr Liang Zhou for helpful discussions. This study was funded by the Chinese National Natural Science Foundation, grant no. 81030010. 


\section{References}

1. El-Serag HB and Rudolph KL: Hepatocellular carcinoma: epidemiology and molecular carcinogenesis. Gastroenterology 132 2557-2576, 2007

2. Farazi PA and DePinho RA: Hepatocellular carcinoma pathogenesis: from genes to environment. Nat Rev Cancer 6: 674-687, 2006.

3. Parkin DM, Bray FI and Devesa SS: Cancer burden in the year 2000. The global picture. Eur J Cancer 37 (Suppl 8): S4-S66, 2001.

4. Ahn JY, Jung EY, Kwun HJ, Lee CW, Sung YC and Jang KL: Dual effects of hepatitis B virus X protein on the regulation of cell-cycle control depending on the status of cellular p53. J Gen Virol 83: 2765-2772, 2002.

5. Zhang JL, Zhao WG, Wu KL, et al: Human hepatitis B virus $\mathrm{X}$ protein promotes cell proliferation and inhibits cell apoptosis through interacting with a serine protease Hepsin. Arch Virol 150: 721-741, 2005.

6. Kang DC, Su ZZ, Sarkar D, Emdad L, Volsky DJ and Fisher PB Cloning and characterization of HIV-1-inducible astrocyte elevated gene-1, AEG-1. Gene 353: 8-15, 2005.

7. Li J, Zhang N, Song LB, et al: Astrocyte elevated gene-1 is a novel prognostic marker for breast cancer progression and overall patient survival. Clin Cancer Res 14: 3319-3326, 2008.

8. Li C, Li R, Song H, et al: Significance of AEG-1 expression in correlation with VEGF, microvessel density and clinicopathological characteristics in triple-negative breast cancer. J Surg Oncol 103: 184-192, 2010.

9. Li J, Yang L, Song L, et al: Astrocyte elevated gene-1 is a proliferation promoter in breast cancer via suppressing transcriptional factor FOXO1. Oncogene 28: 3188-3196, 2009.

10. Hu G, Chong RA, Yang Q, et al: MTDH activation by $8 \mathrm{q} 22$ genomic gain promotes chemoresistance and metastasis of poorprognosis breast cancer. Cancer Cell 15: 9-20, 2009.

11. Baumann BC and Dorsey JF: Astrocyte-elevated gene-1 (AEG-1): glioblastoma's helping hand during times of hypoxia and glucose deprivation? Cancer Biol Ther 11: 40-42, 2011.

12. Noch E, Bookland M and Khalili K: Astrocyte-elevated gene-1 (AEG-1) induction by hypoxia and glucose deprivation in glioblastoma. Cancer Biol Ther 11: 32-39, 2011.

13. Xia Z, Zhang N, Jin H, Yu Z, Xu G and Huang Z: Clinical significance of astrocyte elevated gene-1 expression in human oligodendrogliomas. Clin Neurol Neurosurg 112: 413-419, 2010.

14. Lee SG, Jeon HY, Su ZZ, et al: Astrocyte elevated gene-1 contributes to the pathogenesis of neuroblastoma. Oncogene 28: 2476-2484, 2009.

15. Liu H, Song X, Liu C, Xie L, Wei L and Sun R: Knockdown of astrocyte elevated gene-1 inhibits proliferation and enhancing chemo-sensitivity to cisplatin or doxorubicin in neuroblastoma cells. J Exp Clin Cancer Res 28: 19, 2009.

16. $\mathrm{Xu} \mathrm{J}, \mathrm{Wu} \mathrm{H}, \mathrm{He} \mathrm{Y}$, et al: Astrocyte-elevated gene-1 overexpression is associated with poor prognosis in gastric cancer. Med Oncol 28: 455-462, 2011.

17. Song H, Li C, Li R and Geng J: Prognostic significance of AEG-1 expression in colorectal carcinoma. Int J Colorectal Dis 25 1201-1209, 2010.

18. Wang F, Ke ZF, Sun SJ, et al: Oncogenic roles of astrocyte elevated gene-1 (AEG-1) in osteosarcoma progression and prognosis. Cancer Biol Ther 12: 539-548, 2011.

19. Sun W, Fan YZ, Xi H, Lu XS, Ye C and Zhang JT:Astrocyte elevated gene-1 overexpression in human primary gallbladder carcinomas: an unfavorable and independent prognostic factor. Oncol Rep 26: 1133-1142, 2011.

20. Nohata N, Hanazawa T, Kikkawa N, et al: Tumor suppressive microRNA-375 regulates oncogene AEG-1/MTDH in head and neck squamous cell carcinoma (HNSCC). J Hum Genet 56: 595-601, 2011.

21. Meng X, Brachova P, Yang S, et al: Knockdown of MTDH sensitizes endometrial cancer cells to cell death induction by death receptor ligand TRAIL and HDAC inhibitor LBH589 co-treatment. PLoS One 6: e20920, 2011.

22. Meng F, Luo C, Ma L, Hu Y and Lou G: Clinical significance of astrocyte elevated gene-1 expression in human epithelial ovarian carcinoma. Int J Gynecol Pathol 30: 145-150, 2011.
23. Yu C, Chen $\mathrm{K}$, Zheng $\mathrm{H}$, et al: Overexpression of astrocyte elevated gene-1 (AEG-1) is associated with esophageal squamous cell carcinoma (ESCC) progression and pathogenesis. Carcinogenesis 30: 894-901, 2009.

24. Song L, Li W, Zhang H, et al: Over-expression of AEG-1 significantly associates with tumour aggressiveness and poor prognosis in human non-small cell lung cancer. J Pathol 219: 317-326, 2009.

25. Yoo BK, Emdad L, Su ZZ, et al: Astrocyte elevated gene-1 regulates hepatocellular carcinoma development and progression. J Clin Invest 119: 465-477, 2009.

26. Lee SG, Su ZZ, Emdad L, Sarkar D and Fisher PB: Astrocyte elevated gene-1 (AEG-1) is a target gene of oncogenic Ha-ras requiring phosphatidylinositol 3-kinase and c-Myc. Proc Natl Acad Sci USA 103: 17390-17395, 2006.

27. Emdad L, Sarkar D, Su ZZ, et al: Astrocyte elevated gene-1 recent insights into a novel gene involved in tumor progression, metastasis and neurodegeneration. Pharmacol Ther 114: 155-170, 2007.

28. Kikuno N, Shiina H, Urakami S, et al: Knockdown of astrocyteelevated gene-1 inhibits prostate cancer progression through upregulation of FOXO3a activity. Oncogene 26: 7647-7655, 2007.

29. Song H, Li C, Lu R, Zhang Y and Geng J: Expression of astrocyte elevated gene-1: a novel marker of the pathogenesis, progression, and poor prognosis for endometrial cancer. Int J Gynecol Cancer 20: 1188-1196, 2010

30. Kao WY, Su CW, Chau GY, Lui WY, Wu CW and Wu JC: A comparison of prognosis between patients with hepatitis B and $\mathrm{C}$ virus-related hepatocellular carcinoma undergoing resection surgery. World J Surg 35: 858-867, 2011.

31. Lee YH and Yun Y: HBx protein of hepatitis B virus activates Jak1-STAT signaling. J Biol Chem 273: 25510-25515, 1998.

32. Cha MY, Kim CM, Park YM and Ryu WS: Hepatitis B virus $\mathrm{X}$ protein is essential for the activation of Wnt/beta-catenin signaling in hepatoma cells. Hepatology 39: 1683-1693, 2004

33. Ding Q, Xia W, Liu JC, et al: Erk associates with and primes GSK-3beta for its inactivation resulting in upregulation of betacatenin. Mol Cell 19: 159-170, 2005.

34. Chin R, Nachbur U, Earnest-Silveira L, et al: Dysregulation of hepatocyte cell cycle and cell viability by hepatitis B virus. Virus Res 147: 7-16, 2010.

35. Chin R, Earnest-Silveira L, Koeberlein B, et al: Failure of lamivudine to reverse hepatitis $B$ virus-associated changes in ERK, Akt and cell cycle regulatory proteins. Antivir Ther 13: 221-230, 2008.

36. Chin R, Earnest-Silveira L, Koeberlein B, et al: Modulation of MAPK pathways and cell cycle by replicating hepatitis $B$ virus: factors contributing to hepatocarcinogenesis. J Hepatol 47: 325-337, 2007.

37. Kim HR, Lee SH and Jung G: The hepatitis B viral X protein activates NF-kappaB signaling pathway through the up-regulation of TBK1. FEBS Lett 584: 525-530, 2010.

38. Tanaka Y, Kanai F, Ichimura T, et al: The hepatitis B virus X protein enhances AP-1 activation through interaction with Jab1. Oncogene 25: 633-642, 2006.

39. Chung TW, Lee YC and Kim CH: Hepatitis B viral HBx induces matrix metalloproteinase-9 gene expression through activation of ERK and PI-3K/AKT pathways: involvement of invasive potential. FASEB J 18: 1123-1125, 2004.

40. Lee SG, Su ZZ, Emdad L, Sarkar D, Franke TF and Fisher PB: Astrocyte elevated gene-1 activates cell survival pathways through PI3K-Akt signaling. Oncogene 27: 1114-1121, 2008.

41. Sarkar D, Park ES, Emdad L, Lee SG, Su ZZ and Fisher PB: Molecular basis of nuclear factor-kappaB activation by astrocyte elevated gene-1. Cancer Res 68: 1478-1484, 2008.

42. Emdad L, Sarkar D, Su ZZ, et al: Activation of the nuclear factor kappaB pathway by astrocyte elevated gene-1: implications for tumor progression and metastasis. Cancer Res 66: 1509-1516, 2006.

43. Su P, Zhang Q and Yang Q: Immunohistochemical analysis of Metadherin in proliferative and cancerous breast tissue. Diagn Pathol 5: 38, 2010.

44. Yoo BK, Gredler R, Vozhilla N, et al: Identification of genes conferring resistance to 5-fluorouracil. Proc Natl Acad Sci USA 106: 12938-12943, 2009. 